This is an author produced version of a paper published in Nature Biotechnology. This paper has been peer-reviewed but does not include the final journal pagination.

Citation for the published paper:

Erikson, O., and Hertzberg, M. and Näsholm, T. (2004) A conditional marker gene allowing both positive and negative selection in plants.

Nature Biotechnology. Volume 22: issue 4, pages 455-458. http://dx.doi.org/10.1038/nbt946

Access to the published version may require journal subscription.

Published with permission from: Nature Publishing Group.

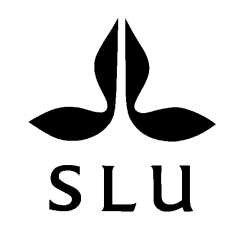

Epsilon Open Archive http://epsilon.slu.se 


\section{A conditional marker gene allowing both positive and negative}

selection in plants

Oskar Erikson ${ }^{1}$, Magnus Hertzberg ${ }^{1,2} \&$ Torgny Näsholm ${ }^{1}$

${ }^{1}$ Umeå Plant Science Centre, Department of Forest Genetics and Plant Physiology, SLU, SE-901 83 Umeå, Sweden

${ }^{2}$ Present address: SweTree Technologies, Uminova Science Park, P.O. Box 7981, SE907 19, Umeå, Sweden

Correspondence should be addressed to T.N. (torgny.nasholm@genfys.slu.se)

Selectable markers enable transgenic plants or cells to be identified after transformation and can be divided into positive and negative markers, conferring a selective advantage or disadvantage, respectively, on the transformed plant or cell. We present a novel marker-gene, dao1 encoding Damino acid oxidase (DAAO, EC 1.4.3.3), that can be used for either positive or negative selection, depending on the substrate. DAAO catalyses the oxidative deamination of a range of $\mathrm{D}$-amino acids $^{1}$ and selection is based on differences in the toxicity displayed by different D-amino acids and their metabolites to plants. Thus, D-alanine and D-serine are toxic to plants, but are metabolised by DAAO into non-toxic products, whereas D-isoleucine and D-valine have low toxicity, but are metabolised into the toxic keto acids 3-methyl-2-oxopentanoate and 3methyl-2-oxobutanoate, respectively. Hence, both positive and negative selection is possible with the same marker gene. The marker has been successfully 
established in Arabidopsis thaliana, and proven to be versatile, rapidly yielding unambiguous results, and allowing selection immediately after germination.

The ability to metabolise D-amino acids is widespread in prokaryotes and many eukaryotes $^{2}$, but current information suggests that D-amino acid metabolism is severely restricted in plants. However, studies of amino acid transporters from plants have shown that several of these proteins may mediate the transport of both $\mathrm{L}-$ and D-enantiomers of amino acids, although the latter usually at lower rates ${ }^{3,4}$. These findings imply that plants are capable of absorbing D-amino acids but have a restricted capacity for D-amino acid metabolism. There are several routes of Damino acid catabolism, one of the most common being oxidative deamination ${ }^{2}$. Damino acid oxidase is a well-characterized enzyme, and both its crystal structure and its catalytic mechanism have been determined by high-resolution $\mathrm{x}$-ray spectroscopy ${ }^{5}$. It is a flavoenzyme located in the peroxisome, and its recognised function in animals is detoxification of $\mathrm{D}$-amino acids ${ }^{2}$. In addition, it gives yeasts the ability to use D-amino acids for growth ${ }^{6}$. D-Amino acid oxidases from several different species have been characterized and shown to differ slightly in substrate affinities $^{7}$, but in general they display broad substrate specificity, oxidatively deaminating all D-amino acids except D-glutamate and D-aspartate ${ }^{2}$. D-amino acid oxidase activity is found in many eucaryotes ${ }^{2}$, but there is no report of DAAO activity in plants.

The low capacity for D-amino acid metabolism in plants has major consequences for the way plants respond to D-amino acids. For instance, our studies of growth responses of $A$. thaliana to $\mathrm{D}-$ serine and $\mathrm{D}$-alanine show that these compounds may 
inhibit growth even at quite low concentrations (Fig. 1a, b). On the other hand, some D-amino acids, like D-valine and D-isoleucine, have minor effects on plant growth (Fig. 1c, d). We therefore hypothesized that the toxicity of D-serine and D-alanine could be alleviated by the insertion of a gene encoding a D-amino acid metabolising enzyme. To test this hypothesis, we transformed wild type $A$. thaliana with the daol gene from the yeast Rhodotorula gracilis under the control of the CaMV 35S promoter. Exposure of this transgenic plant to D-alanine or D-serine showed that it could detoxify both of these D-amino acids (Fig. 1a, b). On the other hand, D-valine and D-isoleucine, which are not toxic to wild-type plants, have a strong negative influence on the growth of the DAAO expressing plants (Fig. 1c, d). The findings that DAAO expression mitigated the toxicity of D-serine and D-alanine, but induced metabolic changes that made D-isoleucine and D-valine toxic suggest that the enzyme could provide a substrate-dependent, dual-function, selectable marker in plants.

To test this idea we germinated $\mathrm{T}_{1}$ seeds on different selective media. The T-DNA contained both 35S::daol and pNos::nptII, allowing D-amino acid and kanamycin selection to be compared in the same lot of seeds. $T_{1}$ seeds were sown on medium containing kanamycin $\left(50 \mu \mathrm{g} \mathrm{ml}^{-1}\right)$, D-alanine $(3 \mathrm{mM})$ or D-serine $(3 \mathrm{mM})$, and the transformation frequencies found on the different selective media were $2.37 \%, 2.12 \%$ and $1.67 \%$, respectively (Fig. 2a-c). D-Alanine had no negative effect on the transgenic plants, even at a concentration of $30 \mathrm{mM}$, but at this concentration, Dserine induced strong growth inhibition (data not shown). We postulated that the lower number of transgenic plants found after selection on $3 \mathrm{mM}$ D-serine may have been due to the compound slightly inhibiting growth of the transgenic plants at this 
concentration. Further studies using lower concentrations corroborated this conclusion, and efficient selection using D-serine was achieved on concentrations lower than $1 \mathrm{mM}$ (Fig. 1a). Progenies from the transgenic lines selected on D-serine and D-alanine were later confirmed kanamycin resistant, hence ensuring no wild type escapes in selection with either D-serine or D-alanine.

Selection of seedlings on media containing D-alanine or D-serine was very rapid compared to selection on kanamycin. These D-amino acids inhibited growth of wild type plants immediately after the cotyledons of wild type plants had emerged. Therefore, transformants could be distinguished from non-transformed plants directly after germination. The difference between wild type and transgenic plants following D-amino acid selection treatment was unambiguous, with no intermediate phenotypes. In contrast, intermediate phenotypes are common when kanamycin resistance is used as a selection marker (Fig. 2c). Furthermore, wild type seedlings were found to be sensitive to sprayed applications of D-serine and D-alanine. Oneweek-old seedlings were effectively killed when sprayed on three consecutive days with either $50 \mathrm{mM}$ D-serine or D-alanine, although the sensitivity of wild type plants rapidly decreased with age, presumably as the cuticle and leaves became thicker and reduced uptake by the leaves. Transgenic seedlings were resistant to foliar application of D-alanine or D-serine, so selection on soil was possible (Fig. 2d). Transgenic plants grown under D-alanine and D-serine selection conditions developed normally. Early development of transgenic plants from line 3:7, 10:7 and 13:4 was compared with that of wild-type plants by cultivation on vertical agar plates. No differences in biomass, number of leaves, root-length or root architecture were detected for the different sets of plants. Furthermore, growth of soil-cultivated wild-type and 
transgenic plants (line 10:7) displayed no differences regarding total number of rosette leaves, number of inflorescences and number of siliqua after four weeks of growth. Also, the phenotypes of 17 individual $\mathrm{T}_{1}$ lines, which were picked for $\mathrm{T}-$ DNA segregation, were studied and found indistinguishable from wild type when grown on soil. A problem sometimes encountered following selection on antibiotics is the growth lag displayed by transformants. This phenomenon is explained as an inhibitory effect of the antibiotic on the transgenic plants ${ }^{8}$. However, unlike seedlings picked from antibiotic selection plates, when transgenic seedlings were picked from D-amino acid selection plates and transferred to soil, their growth and development were not hampered, even temporarily. A possible reason for this difference is that the DAAO scavenging of D-amino acids may effectively remove the $\mathrm{D}$-amino acid in the plants. Furthermore, D-alanine and D-serine may merely provide additional growth substrates, since their catabolic products are carbon and nitrogen compounds that are central compounds in plant metabolism. Quantification of daol mRNA from six independent D-alanine and D-serine resistant lines showed a range of different expression levels (Fig. 2e). These different expression levels were mirrored in a range of different DAAO activities (Fig. 2f). In spite of these differences in mRNA levels and enzyme activities, no phenotypic variation associated with the D-serine and Dalanine treatment was found, suggesting that the DAAO marker is effective over a range of expression levels.

As described above, D-isoleucine and D-valine were found to inhibit growth of the transgenic plants, but not the wild type plants. Therefore, we tested plants containing the construct described above on two sets of media, one containing D-isoleucine and the other containing D-valine at various concentrations, in order to assess whether 
DAAO could also be used as a negative marker. Unambiguous negative selection was achieved when seeds were sown on either D-isoleucine or D-valine at concentrations greater than $10 \mathrm{mM}$ (Fig. 1c, d). Thirteen individual DAAO expressing lines were tested for their response to D-isoleucine and all of them were effectively killed, whereas wild type plants grew well, with no sign of toxicity. Similar results were obtained for D-valine, although this compound was found to have a moderately negative effect on wild type plants at higher concentrations (Fig. 1d). The keto acid produced in DAAO catabolism of D-isoleucine is the same as that formed when $\mathrm{L}$-isoleucine is metabolised by the endogenous branched chain amino acid transaminase [EC: 2.6.1.42], namely 3-methyl-2-oxopentanoate (Kyoto Encyclopedia of Genes and Genomes, metabolic pathway website, http://www.genome.ad.jp/kegg/metabolism.html) We suggest that the endogenous transaminase may be specific for the L-enantiomer, so the corresponding Denantiomer is not metabolised in wild type plants, but only in DAAO expressing plants. The negative effects of $\mathrm{L}$-isoleucine (but not of the $\mathrm{D}$-form) observed on wild type plants, supports this speculation. Incubation of cell-free extracts from daol transgenic line 10:7 with D-isoleucine and D-valine resulted in 15 fold and 7 fold increases in production of 3-methyl-2-oxopentanoate and 3-methyl-2-oxobutanoate, respectively, compared to extracts of wild type plants. Further, 3-methyl-2oxopentanoate and 3-methyl-2-oxobutanoate proved to impair growth of A. thaliana (see Methods), corroborating the suggestion that these compounds, or products of their metabolism, are responsible for the negative effects of D-isoleucine and Dvaline on the transgenic plants. 
The toxicity of some D-amino acids on organisms is not well understood, and has only occasionally been studied in plants ${ }^{9}$. Apart from $A$. thaliana we have also tested other plant species susceptibility to D-serine, including, poplar, tobacco, barley, maize, tomato and spruce. We found all tested species susceptible to D-serine at concentrations similar to those shown toxic for A. thaliana. A proposed mechanism for $\mathrm{D}$-serine toxicity in bacteria is via competitive inhibition of $\beta$-alanine coupling to pantoic acid, thus inhibiting formation of pantothenic acid ${ }^{10}$. It is possible to alleviate D-serine toxicity in D-serine sensitive strains of E. coli by providing pantothenic acid or $\beta$-alanine in the media, but $\mathrm{D}$-serine toxicity in A. thaliana could not be mitigated using these compounds (see Methods). A second putative cause of D-amino acid toxicity is through competitive binding to tRNA. Knockout studies of D-Tyr-tRNA ${ }^{\text {Tyr }}$ deacylase in E. coli have shown that the toxicity of D-tyrosine increases with lack of deacylase activity ${ }^{11}$, indicating that D-amino acids interfere at the tRNA level. Similar genes to bacterial deacylase have also been identified in A thaliana $^{11}$, corroborating the possibility that the mode of toxic action of $\mathrm{D}$-amino acids might be via competitive binding to tRNA.

The natural occurrence of $\mathrm{D}$-amino acids in plants is generally low, with measurable levels of foremost D-alanine, D-serine, D-glutamine and D-asparagine but no detectable levels of D-valine and D-isoleucine ${ }^{12}$. Hence, the amount and nature of substrates that DAAO may engage under natural conditions would not cause negative effects on plants. In agricultural crops the use of antibiotic and herbicide resistance markers is a matter of public concern ${ }^{13}$, and few markers are available that are not based on these mechanisms ${ }^{14}$. Thus, there is a demand for new markers for both research and commercial crop production Alternatives are being sought for and 
DAAO has the potential to provide one such option. Further, removing the selectable marker once it has served its purpose is sometimes desirable, and use of DAAO in existing techniques ${ }^{15}$ may have advantages over other markers in this respect, since both its insertion and loss can be screened, by positive and negative selection, respectively.

\section{Methods}

Vector construction and plant transformation. DNA and RNA manipulation were performed by standard techniques ${ }^{16}$. The yeast Rhodotorula gracilis was grown in liquid culture containing $30 \mathrm{mM}$ D-alanine to induce the $\mathrm{D}$-amino acid oxidase gene daol. Total RNA was isolated from the yeast and used for cDNA synthesis. The PCR primers 5'-ATTAGATCTTACTACTCGAAGGACGCCATG and 5'ATTAGATCTACAGCCACAATTCCCGCCCTA were used to amplify the daol gene from the cDNA template by PCR. The PCR fragments were sub-cloned into the pGEM $^{\circledR}-\mathrm{T}$ Easy vector (Promega) and subsequently ligated into the BamHI site of the CaMV 35S expression cassette of the binary vector pPCV702kana ${ }^{17}$ giving pPCV702:dao1. The vectors were subjected to restriction analysis and sequencing to check that they contained the correct constructs. A. thaliana ecotype Col-0 plants were then transformed by the floral dip method $^{18}$ with Agrobacterium tumefaciens strain GV3101::pMP110 RK carrying the vector described above. Transgenic $\mathrm{T}_{1}$ plants were selected on medium containing kanamycin $\left(50 \mu \mathrm{g} \mathrm{ml} \mathrm{l}^{-1}\right)$. Lines containing a single T-DNA insertion locus were selected by statistical analysis of T-DNA segregation in the $\mathrm{T}_{2}$ population that germinated on kanamycin-containing medium. Plants with a single locus of inserted T-DNA were grown and self-fertilized. Homozygous $T_{3}$ seed stocks were then identified by analysing $T-$ DNA segregation in 
$\mathrm{T}_{3}$ progenies and confirmed to be expressing the introduced gene by northern blot analyses.

Selection analysis. $T_{1}$ seeds were surface-sterilized and sown in Petri plates that were sealed with gas-permeable tape. The growth medium was half strength $\mathrm{MS}^{19}$ with $0.5 \% \mathrm{wt} / \mathrm{vol}$ sucrose and $0.8 \% \mathrm{wt} / \mathrm{vol}$ agar, plus $3 \mathrm{mM}$ D-alanine, $3 \mathrm{mM}$ D-serine or $50 \mu \mathrm{g} \mathrm{ml}^{-1}$ kanamycin as the selective agent. Plants were grown for five days following germination with a $16 \mathrm{~h}$ photoperiod at $24{ }^{\circ} \mathrm{C}$. To evaluate the selection efficiency on different substrates, $2,074,1,914$ and $1,810 \mathrm{~T}_{1}$ seeds were sown on $\mathrm{D}$ alanine, D-serine and kanamycin selective plates, respectively, and the number of surviving seedlings was counted (44, 32 and 43, respectively).

Toxicity studies. To evaluate the toxic action of 3-methyl-2-oxopentanoate and 3methyl-2-oxobutanoate, wild type plants were sown on two sets of half strength MS agar plates, each containing one of the compounds in a range of concentrations $(0.01-$ $10 \mathrm{mM}$ ). Plants were slightly affected by $3-$ methyl-2-oxopentanoate at $0.1 \mathrm{mM}$, and total growth inhibition was observed at $1 \mathrm{mM}$. For 3-methyl-2-oxobutanoate, $5 \mathrm{mM}$ was required for complete inhibition (data not shown). Further, several attempts were made to probe the nature of D-serine's toxicity. In accordance with studies on $E$. $\operatorname{coli}^{10}$, we tried to rescue wild type $A$. thaliana grown on lethal concentrations of Dserine through amendments with five potential inhibitors of D-serine toxicity (Lserine, $\mathrm{Ca}$-pantothenate, $\beta$-alanine, leucine and threonine) added both separately and in combinations in a very wide range of concentrations $\left(0.001-50 \mu \mathrm{g} \mathrm{ml}^{-1}\right)$, without any success. 
Enzyme assays. Soluble proteins were extracted by extracting $0.1 \mathrm{~g}$ samples of plant material that had been finely pulverized in a $1.5 \mathrm{ml}$ eppendorf tube in $1 \mathrm{ml}$ of $0.1 \mathrm{M}$ potassium phosphate buffer, $\mathrm{pH}$ 8. D- amino acid oxidase activity was then assayed as follows. Reaction mixtures were prepared containing 2,120 $\mu \mathrm{l}$ of $0.1 \mathrm{M}$ potassium phosphate buffer ( $\mathrm{pH} 8), 80 \mu 1$ of crude protein extract and $100 \mu \mathrm{l}$ of $0.3 \mathrm{M} \mathrm{D}-$ alanine. The samples were incubated for 2 hours at $30^{\circ} \mathrm{C}$. The enzyme activity was then assessed, by measuring the increase in absorbance at $220 \mathrm{~nm}\left(\Delta \mathrm{E}=1,090 \mathrm{M}^{-1}\right.$ $\mathrm{cm}^{-1}$ ) associated with the conversion of D-alanine to pyruvate, after transferring the test tubes to boiling water for $10 \mathrm{~min}$ to stop the reaction. In control reactions Dalanine was added immediately before boiling. One unit of DAAO activity is defined as the turnover of one micromole of substrate per min, and activity was expressed per gram plant biomass (fresh weight). The breakdown of D-isoleucine and D-valine in DAAO incubations, and the associated production of 3-methyl-2-oxopentanoate and 3-methyl-2-oxobutanoate, were analysed by HPLC ${ }^{20}$. In other respects the reactions were performed as described above.

\section{Acknowledgements}

We would like to acknowledge Margareta Zetherström for help with HPLC analyses and several colleagues for comments on earlier versions of this manuscript. Grants from The Swedish Council for Environment, Agricultural Sciences and Spatial planning, the Kempe foundation, the Wallenberg foundation and Carl Tryggers Stiftelse for financial support to T.N. is gratefully acknowledged. 


\section{References}

1. Alonso, J. et al. D-Amino acid oxidase gene from Rhodotorula gracilis (Rhodosporidium toruloides) ATCC 26217. Microbiol. 144, 1095-1101 (1998).

2. Pilone, M.S. D-Amino acid oxidase: new findings. Cell. Mol. Life. Sci. 57, 17321747 (2000).

3. Frommer, W. B, Hummel, S, Unseld, M \& Ninnemann, O. Seed and vascular expression of a high-affinity transporter for cationic amino acids in Arabidopsis. Proc. Natl. Acad. Sci. USA 92, 12036-12040 (1995).

4. Boorer, K.J., et al. Kinetics and specificity of a $\mathrm{H}^{+}$/amino acid transporter from Arabidopsis thaliana. J. Biol. Chem. 271, 2213-2220 (1996).

5. Umhau, S. et al. The $\mathrm{x}$-ray structure of D-amino acid oxidase at very high resolution identifies the chemical mechanism of flavin-dependent substrate dehydrogenation. Proc. Natl. Acad. Sci. USA 97, 12463-12468 (2000).

6.Yurimoto, H., Hasegawa, T., Sakai, Y. \& Kato, N. Physiological role of the Damino acid oxidase gene DAO1, in carbon and nitrogen metabolism in the methylotrophic yeast Candida boidini. Yeast. 16, 1217-1227 (2000).

7. Gabler, M., Hensel, M. \& Fischer, L. Detection and substrate selectivity of new microbial D-amino acid oxidases. Enzyme Microb. Techno. 27, 605-611 (2000). 8.Lindsey, K. \& Gallois, P. Transformation of sugar beet (Beta vulgaris) by Agrobacterium tumefaciens. J. Exp. Bot. 41, 529-536 (1990).

9. Gamburg, K. Z \& Rekoslavskaya, N. I. Formation and function of D-amino acids in plants. Translated from Fiziologiya Rastenii 38, 1236-1246 (1991).

10. Cosloy, S. D. \& McFall, E. Metabolism of D-serine in Escherichia coli $\mathrm{K}-12$ : Mechanism of growth inhibition. J. Bacteriol. 114, 685-694 (1973). 
11. Soutourina, J., Plateau, P., Delort, F., Peirotes, A. \& Blanquet, S. Functional characterization of the D-Tyr-tRNA ${ }^{\text {Tyr }}$ deacylase from Escherichia coli. J. Biol. Chem. 274, 19109-19114 (1996).

12. Bruckner, H \& Westhauser, T. Chromatographic determination of L-and Damino acids in plants. Amino acids 24, 43-55 (2003).

13. Kuiper, H.A, Kleter, G.A., Noteborn, H.P.J.M. \& Kok, E.J. Assessment of the food safety issues related to genetically modified foods. Plant J. 27, 503-528 (2001). 14. Hare, P. \& Chua, NH. Excision of selectable marker genes from transgenic plants. Nat. Biotechnol. 20, 575-580 (2002).

15. Gleave, A.P., Mitra, D.S., Mudge, S.R. \& Morris, B.A. Selectable marker-free transgenic plants without sexual crossing: transient expression of cre recombinase and use of a conditional lethal dominant gene. Plant Mol. Biol. 40, 223-235 (1999). 16. Sambrook, J. \& Russell, D. W. in Molecular cloning: a laboratory manual $3^{\text {rd }}$ ed. (Cold Spring Harbor Laboratory Press, New York, 2001).

17. Koncz, C. \& Schell, J. The promoter of T-DNA gene 5 controls the tissuespecific expression of chimaeric genes carried by a novel type of vector. Mol. Gen. Genet. 204, 383-396 (1986).

18. Clough, S. J. \& Bent, A. F. Floral dip: a simplified method for Agrobacteriummediated transformation of Arabidopsis thaliana. Plant J. 16, 735-743 (1998). 19. Murashige, T. \& Skoog, F. A revised medium for rapid growth and bioassay with tobacco tissue cultures. Physiol. Plant. 15, 310-313 (1962).

20. Schadewaldt, P., Wendel, U. \& Hammen, HW. Determination of R- and S-3methyl-2-oxopentanoate enantiomers in human plasma: suitable method for label enrichment analysis. J. Chrom. B. 682, 209-218 (1996). 


\section{Figures}

Figure 1. D-amino acid dose-response curves of daol transgenic and wild type Arabidopsis. (a-d) Growth of daol transgenic line 3:7 (•), 10:7 (•), 13:4 (ی) and wild type ( $($ ) plants, in fresh weight per plant, on media containing various concentrations of D-serine, D-alanine, D-isoleucine and D-valine in half strength MS with $0.5 \% \mathrm{wt} / \mathrm{vol}$ sucrose and $0.8 \% \mathrm{wt} / \mathrm{vol}$ agar. Please note the different concentration ranges used for the different D-amino acids. The plants were grown for 10 days following germination under $16 \mathrm{~h}$ photoperiods at $24{ }^{\circ} \mathrm{C} ; \mathrm{n}=10 \pm \mathrm{SE}$, except for plants grown on D-isoleucine, where smaller Petri dishes were used, $(n=6 \pm S E)$, (e-h) and (i-l) Photographs of daol transgenic line 10:7 and wild type plants, respectively, grown for 10 days on the highest concentrations of the D-amino acids shown in the respective graphs above. All pictures have the same magnification.

Figure 2. Selection of primary transformants with the DAAO marker. DAAO $\mathrm{T}_{1}$ seedlings on media containing (a) $3 \mathrm{mM}$ D-alanine, (b) $3 \mathrm{mM} \mathrm{D-serine} \mathrm{and} \mathrm{(c)} 50 \mu \mathrm{g}$ $\mathrm{ml}^{-1}$ kanamycin. Seeds were surface sterilised and sown on half strength MS plates with $0.5 \% \mathrm{wt} / \mathrm{vol}$ sucrose, $0.8 \% \mathrm{wt} / \mathrm{vol}$ agar and the respective selective compound, then grown for five days following germination under $16 \mathrm{~h}$ photoperiods at $24{ }^{\circ} \mathrm{C}$. (d) DAAO transgenic plants grown on soil photographed following selection by spraying with (i) D-alanine and (ii) D-serine, and wild type plants sprayed with (iii) D-alanine and (iv) D-serine. Eight seeds were sown per plot and treatment, on soil, and grown for seven days following germination before applying the selective treatment, which consisted of spraying with aqueous $50 \mathrm{mM}$ solutions of D-alanine or D-serine with $0.05 \%$ Tween 80 on three consecutive days. (e) Northern blot analysis of daol mRNA 
levels from six D-serine and D-alanine resistant lines and wild type plants. Ten $\mu \mathrm{g}$ total RNA was loaded per lane and separated on an agarose gel. Ethidium bromidestained total RNA bands are shown as loading controls. (f) D-Amino acid oxidase activity in six transgenic lines and wild type. A unit of DAAO activity is defined as the turnover of one micromole of substrate per min. Bars represent means, $n=3 \pm S E$. 

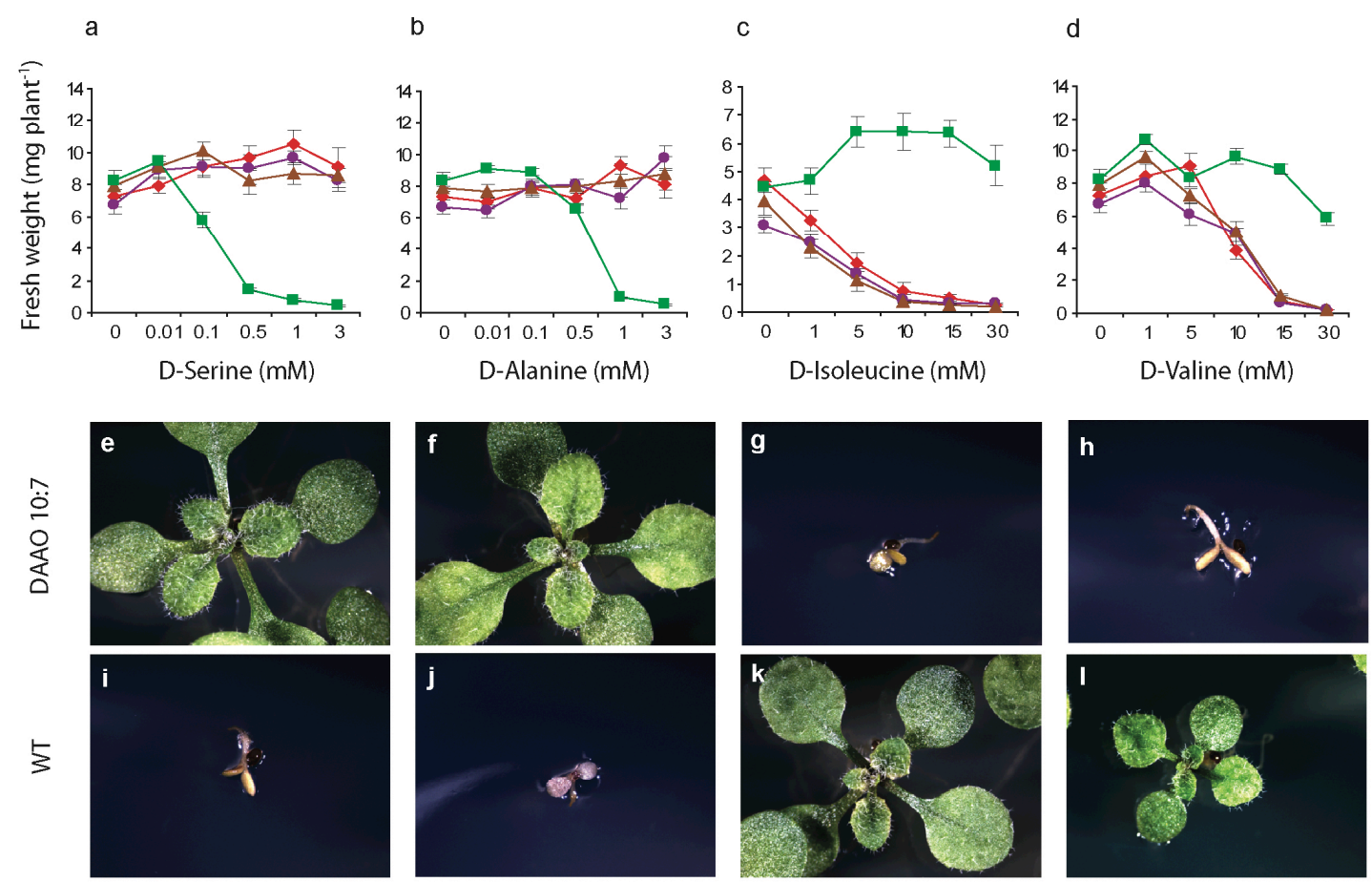


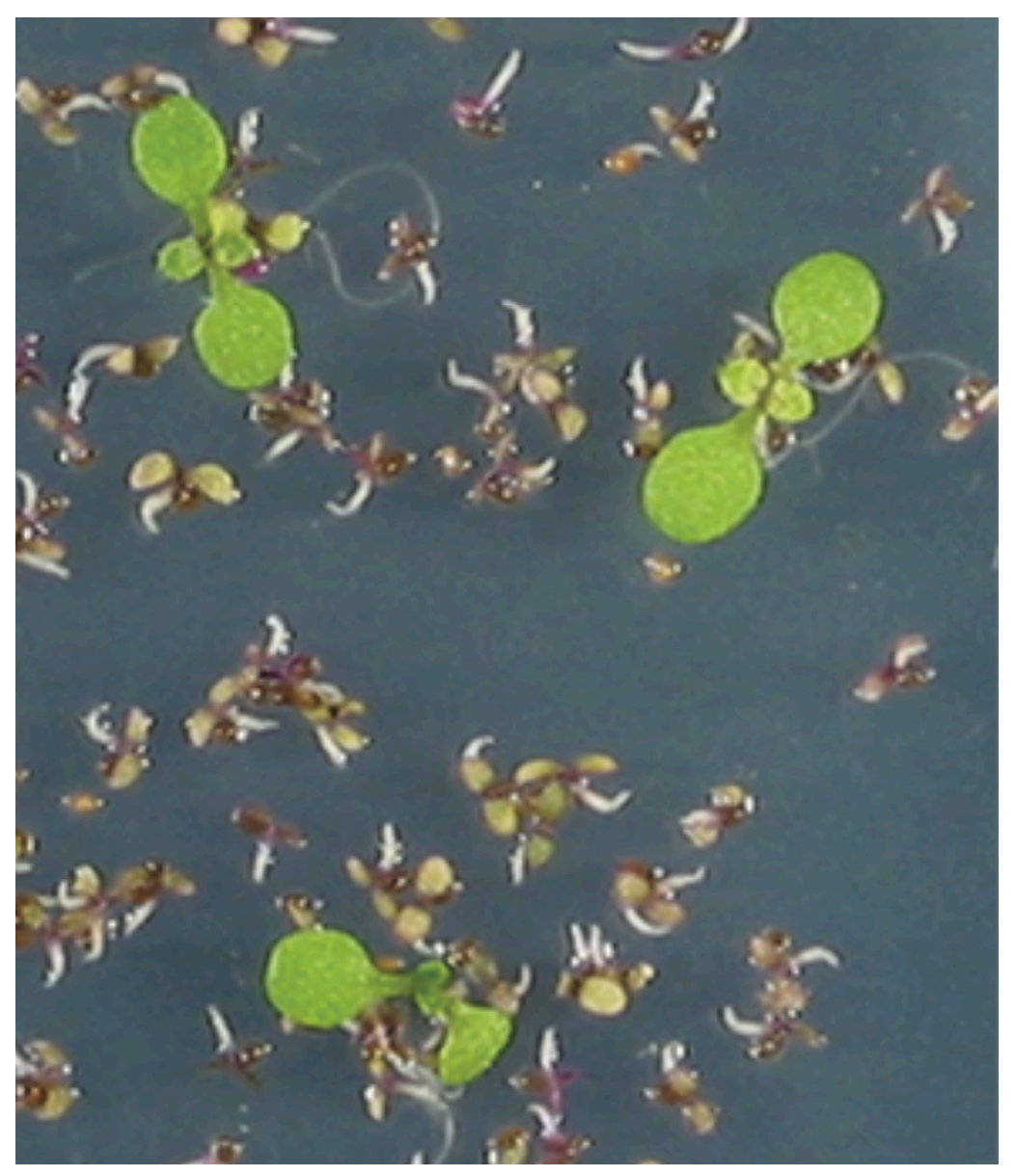

Figure 2a 


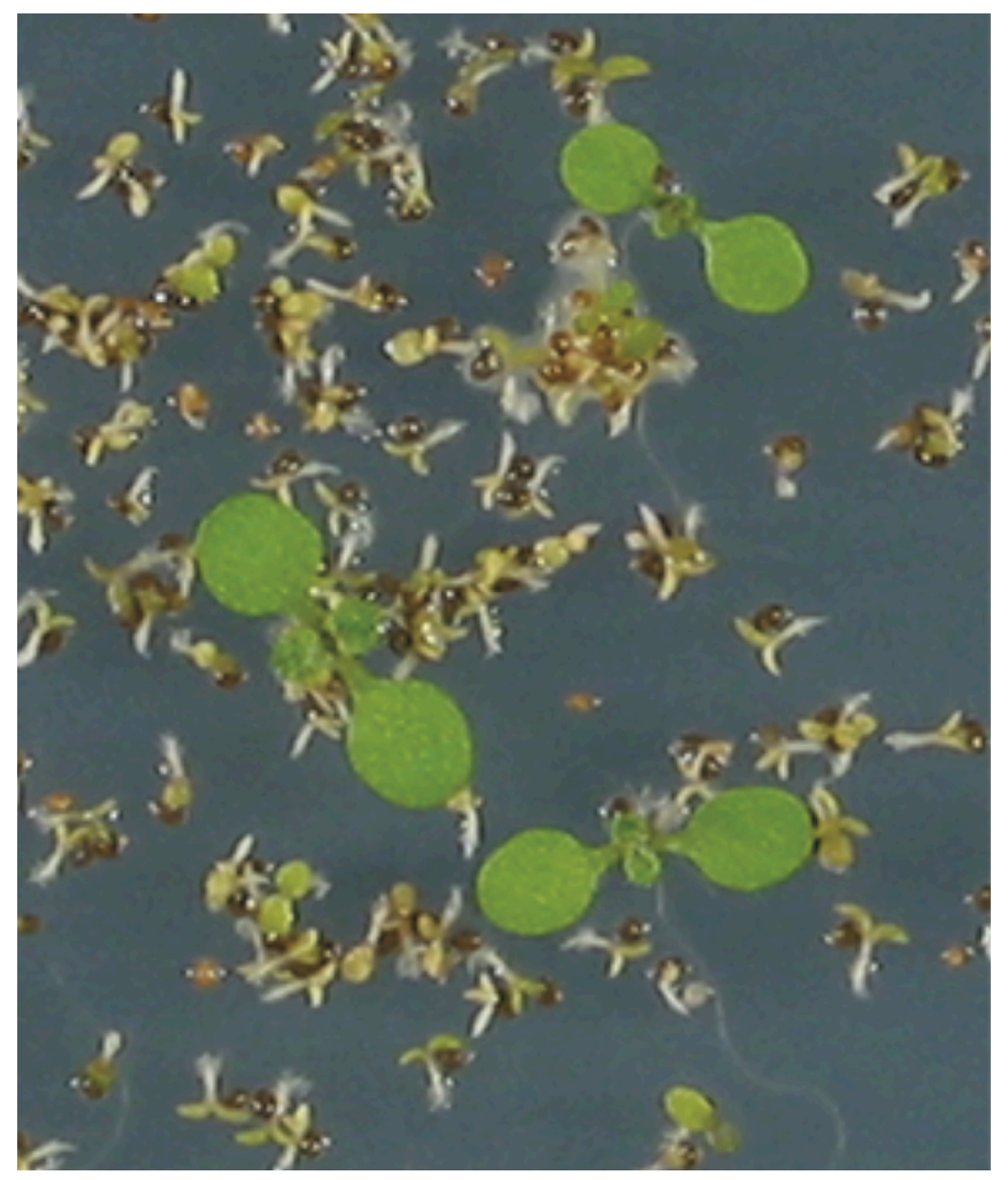

Figure $2 b$ 


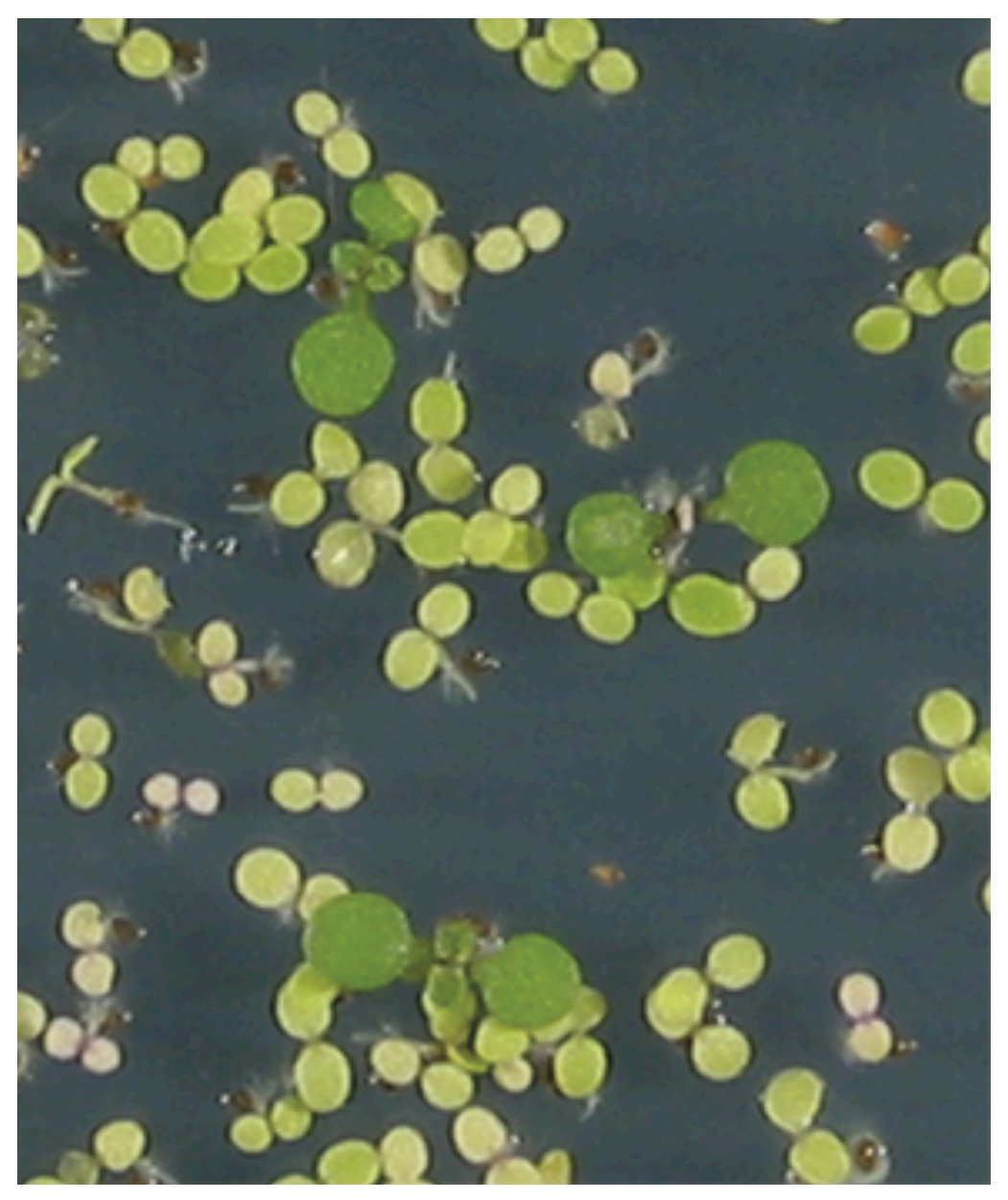

Figure 2c 


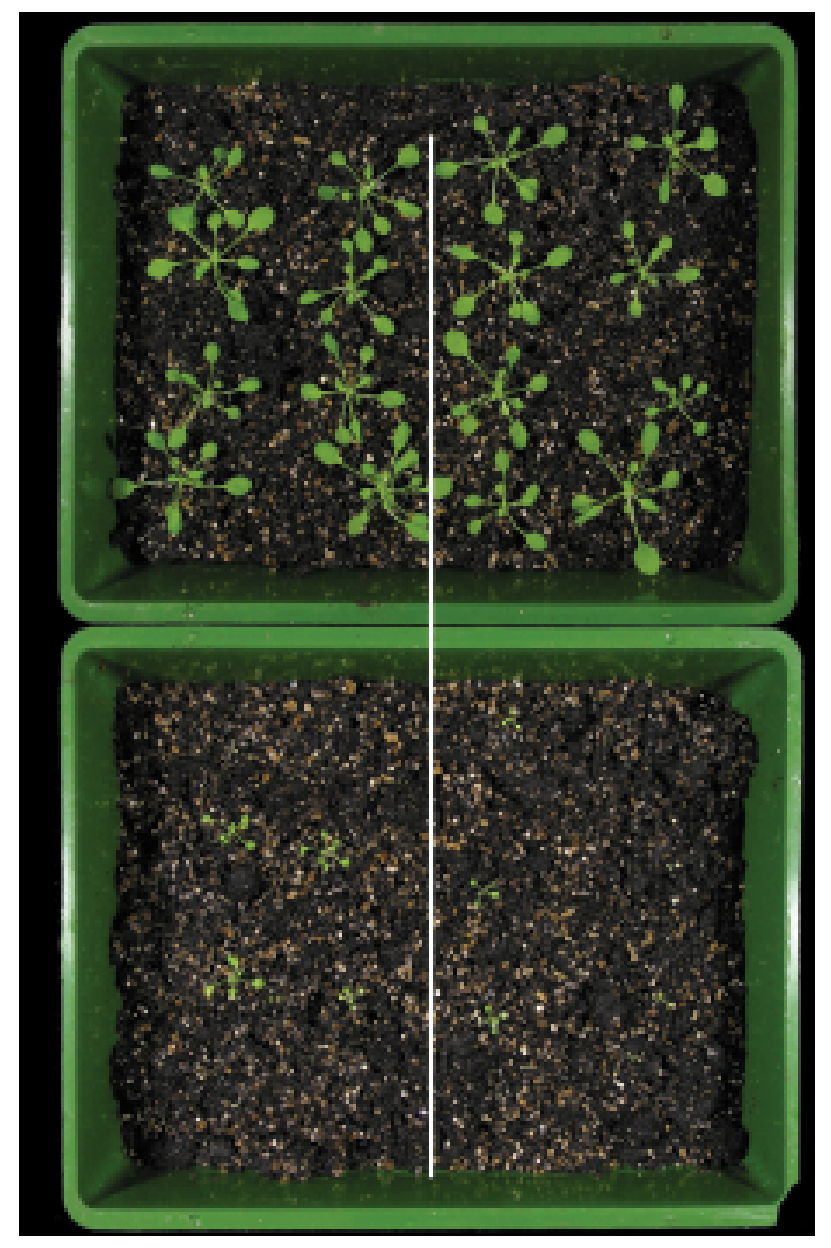

Figure $2 d$ 
dao1

\section{Total RNA}

$$
\begin{array}{llllll}
3: 7 & 4: 7 & 6: 8 & 10: 7 & 12: 8 & 13: 4 \\
\mathrm{Wt}
\end{array}
$$

Figure 2e 


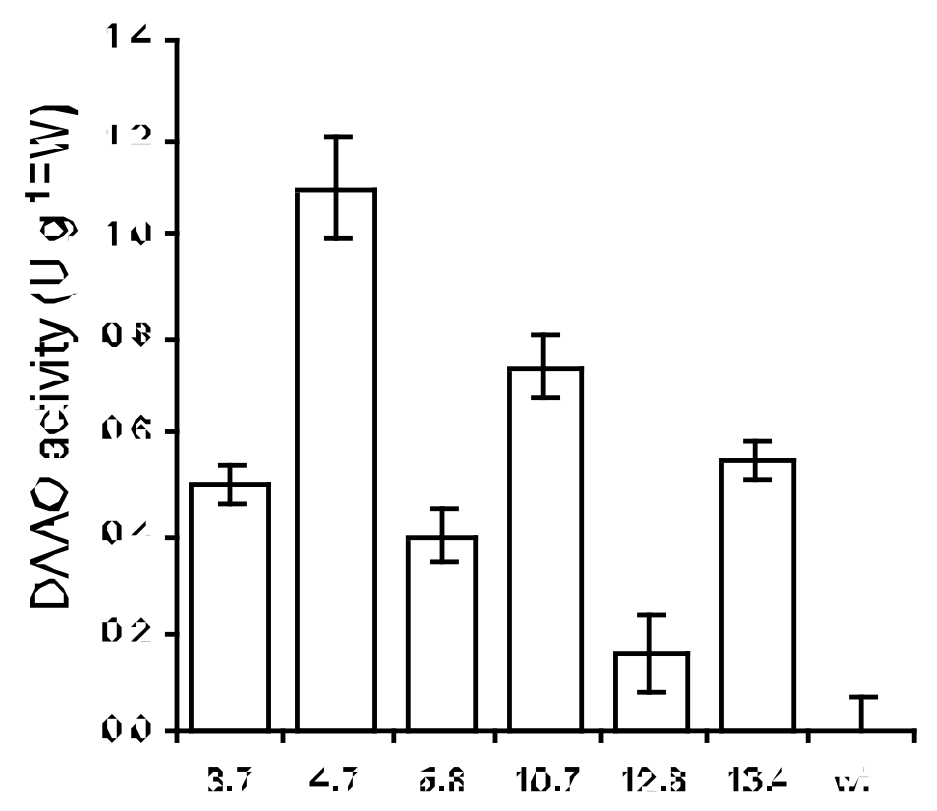

Figure $2 \mathrm{f}$ 